\title{
CONOCIMIENTO Y ACTITUD SOBRE LOS EFECTOS NOCIVOS DE LA RADIACIÓN SOLAR Y PRÁCTICAS DE FOTOPROTECCIÓN EN ESTUDIANTES DE ENFERMERÍA QUE REALIZAN PRÁCTICAS COMUNITARIAS EN LA UNIVERSIDAD NACIONAL SAN LUIS GONZAGA DE ICA, AGOSTO 2011 - SETIEMBRE 2012.
}

\section{Knowledge and attitude on the harmful effects of solar radiation and behavior of nursing students who practice community at the Universidad Nacional San Luis Gonzaga de Ica, August 2011 - September 2012.}

\author{
Flora Martha Huisacayna Diaz ${ }^{1,3 ; a, b, c}$, Yolanda Aguilar Semino ${ }^{1,3 ; a}$, Wendy Malpartida Carrillo ${ }^{2,3 ; a}$. \\ ${ }^{1}$ Facultad de Enfermería. Universidad Nacional San Luis Gonzaga de Ica. Ica, Perú. \\ ${ }^{2}$ Facultad de Enfermería. Universidad Privada San Juan Bautista. Ica, Perú. \\ ${ }^{3}$ Hospital Santa María del Socorro. Ica, Perú. \\ a Lic. En Enfermería, b Enfermera Especialista - Médico Quirúrgico, c Magister en Educación con mención en \\ Administración y planificación en Educación Superior.
}

\section{RESUMEN}

Objetivo: Relacionar el nivel de conocimiento sobre los efectos nocivos de la radiación solar y fotoprotectores con las actitudes y medidas de fotoprotección en estudiantes de enfermería que realizan prácticas comunitarias en la Universidad Nacional San Luis Gonzaga de Ica durante el periodo agosto 2011 - setiembre 2012. Material y Métodos: El estudio fue de nivel aplicativo, tipo cuantitativo, método descriptivo correlacional de corte transversal, la muestra lo constituyeron 93 estudiantes que realizaron sus prácticas en la comunidad, la técnica fue la encuesta auto administrada con tres cuestionarios. Se utilizó como herramienta la estadística descriptiva y los analizadores chi cuadrado y la $\mathrm{t}$ estudent del paquete estadístico S.P.S.S. para un nivel de confianza del $95 \%$ y máximo error permisible del 5\%. Resultados: Setenta de los estudiantes tiene suficiente nivel de conocimiento $(75,27 \%) ; 65(69,9 \%)$ tienen actitudes favorables para evitar los efectos nocivos de la radiación solar y $63(67,74 \%)$ realizan prácticas adecuadas de fotoproteccion. Conclusiones: La prueba de hipótesis afirman relación estadística significativa $(\mathrm{P}<0,005)$ entre el nivel de conocimiento y las actitudes y prácticas de fotoprotección.

PALABRAS CLAVE: Knowledge, attitude, adverse effects, solar radiation, photoprotection practices.

\section{SUMMARY}

Objectives: To relate the level of knowledge on the harmful and photoprotective effects of the solar radiation to the attitudes and photoprotection measures in nursery students, who realize community practices at the Universidad Nacional San Luis Gonzaga de Ica during the period August 2011 September 2012. Material and Methods: The study was of applicative level, quantitative type, descriptive method: correlacional of transverse court, the sample it was constituted by 93 students who did their professional practice in the community, the technology was the survey car administered with three questionnaires. I use as tool the descriptive statistics and the analyzers chi square and her $t$ sstudent of the statistical package S.P.S.S. for a confidence level of $95 \%$ and maximum permissable mistake of $5 \%$. Results: 70 of the students has sufficient level of knowledge $(75.27 \%), 65(69.9 \%)$ has favorable attitudes to avoid the harmful effects of the solar radiation and $63(67.74 \%)$ realizes suitable practices of photoprotection. Conclusions: The test of hypothesis they affirm statistical significant relation $(P<0.005)$ between the level of knowledge and the attitudes and photoprotection practices.

KEY WORDS: Knowledge of solar radiation, attitudes towards the solar radiation, photoprotection measures, photoprotectors, students of infirmary. 


\section{INTRODUCCION}

Según la United Nations Environment Programme (UNEP) el cáncer de piel ocupa el cuarto lugar, aumentando de $2-4 \%$ en 5 años, 18 millones de personas en el mundo han quedado ciegos a causa de catarata atribuibles directamente a la exposición de la radiación ultravioleta (1). La Organización Mundial de la Salud (OMS) refiere que las radiaciones solares ejercen un efecto negativo para la salud, principalmente debido a la relación de estas con determinados tipos de cáncer de piel, envejecimiento prematuro de la piel, cataratas y otras enfermedades oculares (2), estos cambios que se producen en la piel por la exposición crónica a los rayos solares se manifiesta después de 30 años, incluso pueden desarrollarse entre los 20 años aun en personas que viven en zonas muy soleadas por lo que la Academia Norteamericana de Dermatología recomienda un examen completo de la piel anualmente. Se estima que el $45 \%$ de todos los cánceres prevenibles del mundo tiene su origen en la piel, la mayoría de estudios muestran que las tasas se encuentran incrementándose significativamente en todo el mundo, constituyendo un problema de salud pública emergente (3).

El melanoma es un cáncer de la piel que tiene de las consecuencias más perjudiciales para la salud, debido a la gran capacidad que tiene para producir metástasis. La importancia de este cáncer en particular esta, en que durante los últimos 10 años se han incrementado la cantidad de casos, más que algún otro tipo de cáncer. El origen de esta patología se debe a la respuesta de las células cutáneas: melanocito y queratinocito a los rayos UV-B. Estas células son capaces de proliferar por lo que tiene mayor riesgo de conversión maligna. Otros daños para la salud son los que se producen a nivel ocular como la catarata, reducción de la respuesta del sistema inmunitario producido por el quiebre de ADN en los linfocitos originados por los UV
-B que dan del organismo expuesto a diversas enfermedades contagiosas (4).

En el Perú se producen entre tres y cinco mil nuevos casos cada año y la incidencia porcentual de cáncer de piel se elevó de 2 a $4 \%$ en los últimos años como consecuencia a la exposición al sol sin protección. Perú es uno de los 5 países con niveles de radiación ultravioleta más alto del mundo (5), la OMS recuerda tomar medidas inmediatas para protegerse de la radiación ultravioleta.

El objetivo de esta investigación fue relacionar el nivel de conocimiento sobre los efectos nocivos de la radiación solar y fotoprotectores con las actitudes y medidas de fotoprotección en estudiantes que realizan prácticas comunitarias en la UNICA durante el periodo agosto 2011 - setiembre 2012.

\section{MATERIAL Y MÉTODOS}

Este trabajo de investigación es de nivel aplicativo, cuantitativo, correlacional, descriptivo de corte transversal. La muestra lo conforman 93 estudiantes de enfermería que realizan sus prácticas comunitarias. Se utilizó la encuesta como técnica de recolección de datos y como instrumentos tres cuestionarios: Primer cuestionario relacionado a los conocimientos de los estudiantes sobre efectos nocivos de la radiación solar, el Segundo cuestionario sobre las actitudes de los estudiantes hacia los efectos nocivos de la radiación solar y el Tercer cuestionario sobre las prácticas de fotoprotección, dichos cuestionarios fueron validados mediante juicio de expertos investigadores en salud público. Para evaluar la confiabilidad del instrumento se realizó una prueba piloto del $15 \%$ de la muestra total en estudio. Se utilizó como herramienta la estadística descriptiva y los analizadores del chi cuadrado y t de sstudent del paquete estadístico S.P.S.S. para un nivel de confianza del $95 \%$ y máximo error permisible del $5 \%$. 


\section{RESULTADOS}

Setenta de los estudiantes en estudio tienen nivel de conocimiento suficiente sobre efectos de la radiación solar, semáforo solar, bloqueador solar, tiempo de exposición de bloqueador, métodos de protección, 23 con un nivel insuficiente, 65 tienen actitudes favorables al uso de bloqueador correcto, gorro o sombrero de ala ancha, blusa con manga larga, bloqueadores solares, lentes oscuros cerrados lateralmente, búsqueda de sombra, 28 con actitudes desfavorables, 63 realizan prácticas adecuadas de fotoprotección y 30 utilizan prácticas inadecuadas.

TABLA No. 1 - DATOS GENERALES SOBRE EFECTOS NOCIVOS DE LA RADIACION SOLAR EN ESTUDIANTES QUE REALIZAN PRACTICAS COMUNITARIAS

\begin{tabular}{|l|c|c|}
\hline \multicolumn{1}{|c|}{ VARIABLES } & FRECUENCIA & PORCENTAJE \\
\hline Sexo: & 87 & $93.54 \%$ \\
$\quad$ Femenino & 6 & $6.46 \%$ \\
Masculino & & \\
& 54 & $58.1 \%$ \\
Grupo Etareo & 21 & $22.6 \%$ \\
$18 \cdot 19$ & 11 & $11.8 \%$ \\
$20 \cdot 21$ & 7 & $7.5 \%$ \\
$22 \cdot 23$ & & \\
$24 \cdot 25$ & & \\
Nivel de conocimiento sobre efectos nocivos de radiación solar & & \\
Suficiente & 70 & $75.27 \%$ \\
Insuficiente & 23 & $24.73 \%$ \\
Actitudes hacia los efectos nocivos de la radiación solar & & \\
Favorables & 65 & $69.9 \%$ \\
Desfavorables & 28 & $30.1 \%$ \\
& & \\
Practicas de foto protección: tónico y externo & & \\
Adecuadas & & \\
Inadecuadas & 63 & $67.74 \%$ \\
& 30 & $32.26 \%$ \\
\hline
\end{tabular}

Gráfico 1: Nivel de conocimiento sobre efectos nocivos de la radiación solar en estudiantes que realizan prácticas comunitarias.

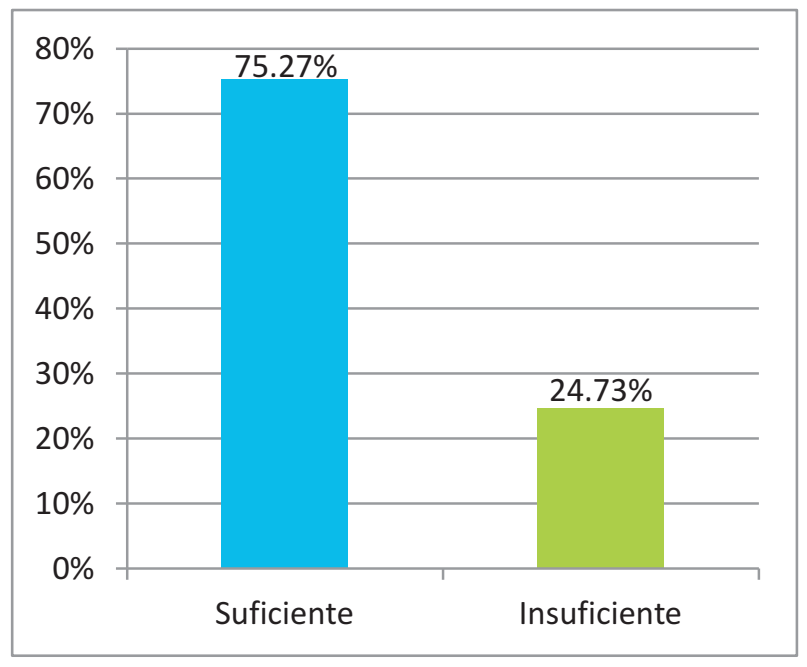

GRAFICO 2: Actitudes hacia los efectos nocivos de la radiación solar en estuantes que realizan prácticas comunitarias.

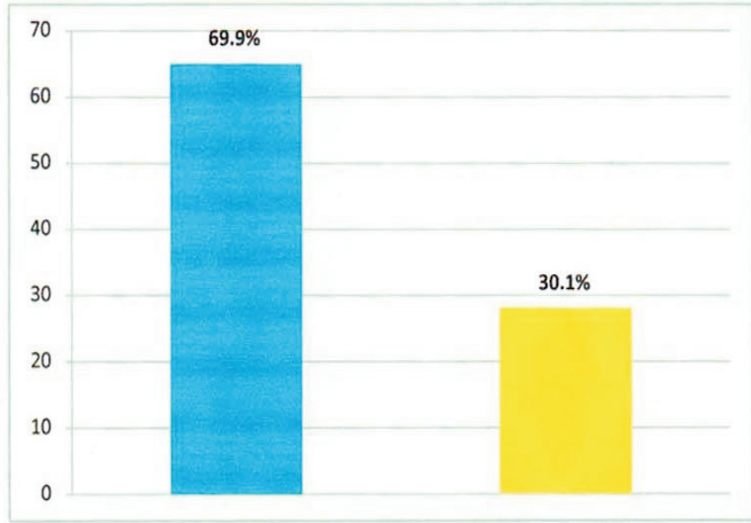

Favorables Desfavorables

GRAFICO 3: Prácticas de fotoproteccion - tópicos y externos en estudiantes que realizan prácticas comunitarias.



PRACTICAS DE FOTOPROTECCION Adecuadas

Inadecuadas

\section{DISCUSIÓN}

Con respecto al nivel de conocimiento sobre los efectos nocivos de la radiación solar y fotoprotectores los hallazgos nos indican que $70(75,27 \%)$ de los estudiantes que realizan prácticas comunitarias tienen suficiente nivel de conocimiento sobre radiación solar (ultravioleta, efectos) y los fotoprotectores tópicos (bloqueador solar) y externos (uso de gorro, ropa de manga larga, gafas, búsqueda de áreas sombreadas) y $23 \quad(24,73 \%)$ tienen insuficiente nivel de conocimiento. Estos resultados contrastan con los hallazgos de Castañeda et al quienes concluyen que el $71 \%$ tienen conocimientos sobre efectos nocivos de radiación solar (6), en el estudio de Rios encontramos iguales resultados el 
$54 \%$ de conocimiento es aceptable (7), así también Thomas considera tener conocimientos aceptables relacionados a los riesgos ocasionados por la radiación solar en un $58 \%$ (8). Respecto a las actitudes para evitar los efectos nocivos de la radiación solar, los hallazgos señalan que el $69.9 \%$ de los estudiantes tienen actitudes favorables, dichos resultados difieren a los hallados por por Ríos, donde concluye que las actitudes de los estudiantes son positivas en un $70 \%$ (7).

Respecto a la práctica de fotoprotección (uso de gorro, lentes, bloqueador solar, vestimenta con magas largas, búsqueda en horas de mayor radiación solar) muestran resultados $67,74 \%$ realizan prácticas adecuadas de fotoprotección, similares hallazgos se encontraron por Hynowit con que el $59.1 \%$ tienen practicas adecuadas (9), así también podemos ver que Salinas indica que el $60 \%$ de los estudiantes tiene un nivel inadecuado de prácticas de fotoprotección (10).

\section{CONCLUSIONES}

Se ha probado que existe relación estadística entre el nivel de conocimiento con las actitudes y el uso de medidas de fotoprotección por parte de los alumnos.

\section{Correspondencia:}

Mg. Flora Martha Huisacayna Dïaz

Correo electrónico:

martha.fhd@hotmail.com

\section{REFERENCIAS BIBLIOGRÁFICAS:}

1. Programa de las naciones unidas para el medio ambiente. Acción por el ozono (Internet). $2^{\circ}$ Edición. Nairobi, Kenya: Editorial PNUMA/ Secretaria de Ozono; 2000. (Citado el 20 de febrero del 2013) Disponible en http://ozone.unep.org/pdfs/ozone-actionsp.pdf

2. Araya MC. Folleto del Programa de Fotoeducación; 2004.
3. El Sayed F, Ammoury A, Nakhle F, Dhaybi R, Marguery MC. Photoprotection in teenagers. Photodermatol Photoimmunos Photomed. 2006; 22:18-21.

4. Lopez G. Protocolo de Montreal. Alianzas exitosas pata la protección de la capa de ozono el caso de Chile. p. 2026,29 .

5. Ccora O, Echeandia A. Evaluación y pronóstico de la radiación ultravioleta B en las ciudades de Lima, Arequipa, Cajamarca y Puno periodo setiembreoctubre 2007. Lima, Perú: Servicio Nacional de Meteorología e Hidrología; 2007.

6. Castañeda J, Torres B. Conocimiento y actitudes de la población mexicana. Tesis. México DF, Mexico; 2006.

7. Rios D. Conocimiento sobre efectos nocivos de la radiación solar en Arequipa. Tesis. Ica, Perú. Universidad San Luis Gonzaga de Ica. 2008.

8. Thomas E. Efecto de la radiación solar sobre los ojos. España:Centro de Investigación, diagnóstico y tratamiento oftalmológico. 2007. p. 37-38.

9. Hynowit M. Conocimiento, actitudes y la práctica personal de fotoproteccion y el auto examen cutáneo en estudiantes de medicina de la Universidad de Boston. Boston, USA: Universidad de Boston .p. 114-120.

10. Salinas J. Nivel de conocimiento sobre los efectos nocivos de la radiación solar y prácticas de medidas de fotoprotección que adoptan para con sus hijos los padres de familia que acuden a los consultorios externos de pediatría del Hospital Regional Honorio Delgado E. Arequipa, 2002. Tesis para optar el título de Bachiller de Medicina. Arequipa, Perú. Universidad Nacional San Agustín, 2002.

Revisado: 03/12/12

Aprobado para publicación: 14/12/12 